\title{
Avaliação estatística da variação da velocidade de propagação de ondas de ultra-som na madeira em presença de defeitos
}

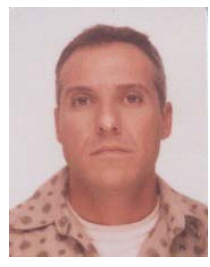

Carlos T. Puccini ${ }^{1}$, Raquel Gonçalves ${ }^{2} \&$ Mariu E. A. Monteiro ${ }^{3}$

\begin{abstract}
1 FEAGRI/UNICAMP. C. P. 6011. Fone: (19) 3788-1050, Fax: (19) 3788-1010. E-mail: puccini@agr.unicamp.br (Foto)
${ }^{2}$ FEAGRI/UNICAMP. C. P. 6011. Fone: (19) 3788-1034, Fax: (19) 3788-1010. E-mail: raquel@agr.unicamp.br

3 IMECC/UNICAMP. E-mail: mariumonteiro@hotmail.com
\end{abstract}

Protocolo $173-5 / 12 / 2001$

\begin{abstract}
Resumo: Os métodos visuais para a detecção de defeitos na madeira, ainda que de grande utilidade, podem ser falhos e exigem a utilização de mão-de-obra relativamente bem treinada. Muitas vezes, a presença de nós, rachaduras ou, ainda, regiões com medula no interior de uma peça serrada, podem ser imperceptíveis na avaliação visual. Um dos grandes avanços obtidos nos últimos anos na caracterização de materiais, tanto do ponto de vista mecânico quanto da qualidade, é a aplicação de técnicas que utilizam a propagação de ondas, destacando-se, dentre elas, o ultra-som. O objetivo deste trabalho é avaliar, estatisticamente, a possibilidade de se utilizar o método do ultra-som na detecção de defeitos em peças de madeira serrada. Para isto, foram utilizadas 180 peças de dimensões nominais: 0,027 $\mathrm{m}$ de espessura; $0,10 \mathrm{~m}$ de largura e $0,25 \mathrm{~m}$ de comprimento, retiradas de vigas de Pinus $s p$ obtidas em serraria da cidade de Campinas. As peças foram ensaiadas na umidade de equilíbrio ao ar. Para a realização dos ensaios utilizouse o equipamento de emissão de ondas de ultra-som marca Steinkamp BP-7 com transdutores de $45 \mathrm{kHz}$. Inicialmente foi realizada uma análise visual das peças e, posteriormente, a determinação da velocidade de propagação das ondas nas mesmas peças. Com os resultados, realizou-se uma análise exploratória das variáveis e obteve-se um modelo de regressão logística visando verificar a relação entre a presença ou não de defeitos e a velocidade de propagação da onda do ultra-som na madeira. Os resultados demonstram uma forte relação entre a velocidade de propagação da onda de ultra-som e os defeitos detectados pela análise visual
\end{abstract}

Palavras-chave: análise visual, ultra-som, defeitos na madeira

\section{Statistical evaluation of the ultrasonic wave velocity variation in defective wood}

\begin{abstract}
Visual methods to detect wood defects, although of great utility, can lead to inaccurate results and demand a specialist to carry it out. The presence of knots, cracks or pith regions inside a sawed piece can be imperceptible in the visual evaluation. One of the greatest progress obtained in the last years in the characterization of materials - not only to determine its mechanical properties but also its quality - is the application of acoustic techniques like the ultrasound. The objective of this study is to evaluate, by statistical analysis, the sensibility of the ultrasonic method for detection of defects in pieces of sawed wood. For the trail, 180 pieces of Pinus $s p$. with $0.027 \mathrm{~m}$ of thickness, $0.10 \mathrm{~m}$ of width and 0.25 of length were used. These pieces tested under air dried condition (approximately $12 \%$ moisture content), were obtained at a saw mill in Campinas. The velocity of ultrasonic waves was measured by Steinkamp BP-7 ultrasound equipment with $45 \mathrm{kHz}$ transducers. Initially the pieces were analyzed by visual method and later the velocity of ultrasonic waves through the wood pieces was measured. An exploratory analysis of the variables was done and a logistic regression model was obtained. The objective was to verify the relationship for the presence or absence of defects in the wood and the velocity of ultrasonic wave propagation. The results demonstrate a strong relationship between the ultrasonic wave propagation and the defects detected by the visual analysis
\end{abstract}

Key words: visual analysis, ultrasound, wood defects 


\section{INTRODUÇÃO}

O uso da madeira para diversas finalidades construtivas é reconhecido e valorizado em diversas partes do mundo. Países como Canadá, França, Suíça, Japão e Estados Unidos, têm investido recursos financeiros e humanos na busca de conhecimento do material bem como de novas técnicas de aplicação.

Um dos grandes avanços obtidos nos últimos anos na caracterização de materiais, tanto do ponto de vista mecânico quanto de qualidade, é a aplicação de técnicas não destrutivas denominadas, internacionalmente, NDT (Non Destructive Testing) e NDE (Non Destructive Evaluation) destacando-se, dentre elas, o uso do ultra-som. Em relação à qualidade da madeira, sobretudo quanto a tipologia e freqüência dos defeitos, no Brasil não existe uma norma de uso generalizado e, sim, normas visando aplicações específicas. Exemplo dessas normas são a NBR 9192/95 (ABNT, 1997) e a Norma para Classificação de madeira serrada de folhosas, do IBDF (IBDF, 1993). Estas normas não descrevem critérios de classificação, mas apenas especificações que definem a aceitação ou rejeição de determinado lote e se baseiam, tão somente, em métodos visuais.

Os métodos visuais, bastante utilizados no passado em normas de classificação, podem ser falhos e exigem a utilização de mão-de-obra relativamente bem treinada. Muitas vezes, a presença de nós, rachaduras ou, ainda, regiões com medula no interior de uma peça serrada, pode ser imperceptíveis na avaliação visual. No caso de peças com presença de madeira juvenil, o método visual é, também, pouco produtivo, pois exigiria grande experiência do avaliador para ser utilizado com sucesso.

A classificação visual é o mais tradicional método sendo bastante utilizada na Comunidade Européia e nos EUA. Este método consiste na análise de peças estruturais por um profissional de larga experiência, visando à detecção de nós, distorção das fibras, presença de fungos e insetos e demais defeitos nas mesmas. A classificação é feita com base no número de defeitos, sendo as classes determinadas pelo Eurocode-5.

A técnica utilizando o ultra-som, é mais recente e se trata de um fenômeno físico que auxilia na determinação da resistência e rigidez de peças de madeira bem como possibilita a detecção de aspectos de qualidade como, por exemplo, nós, medulas etc. Bucur (1995) determinou as equações fundamentais da propagação das ondas de ultra-som na madeira, o que significou um avanço importante nesse campo. O ensaio consiste na emissão de um sinal elétrico por um gerador, que é transformado em pulso de ultra-som por meio de um transdutor. Este pulso atravessa o corpo-de-prova e é recebido pelo transdutor receptor, onde é novamente transformado em sinal elétrico, o qual pode ser visualizado em um osciloscópio. Durante o ensaio é possível medir-se o tempo decorrido entre a saída e a chegada da onda, ou entre a emissão e a recepção. Trata-se de uma técnica muito simples e cuja precisão de leitura é realizada com menos de $1 \%$ de erro.

Objetivou-se com este trabalho, avaliar, estatisticamente, a sensibilidade do método do ultra-som na detecção de defeitos (presença de nós e desvio de fibras) e de características indesejáveis (presença de medula) em peças de madeira para aplicação industrial, visando o estabelecimento de critérios que auxiliem na proposição de um método de classificação para a madeira, utilizando-se o ultra-som. No decorrer do trabalho, além de uma análise exploratória das variáveis utilizadas foi desenvolvido um modelo de regressão logística, visando comprovar a relação entre a presença ou não de defeitos na madeira e a velocidade de propagação da onda de ultrasom. Como análise secundária foram inferidos intervalos de velocidade de onda, nos quais os defeitos estão localizados, construindo-se intervalos de confiança. Os fundamentos utilizados para as avaliações estatísticas e para as interpretações dos resultados fornecidos pelos pacotes computacionais foram baseadas em publicações de Mood (1973), Conover (1980), Meyer (1983) e Agreste (1990).

\section{MATERIAL E MÉTODOS}

\section{Material}

A espécie de madeira adotada foi o Pinus sp. A adoção desta espécie se deu, principalmente, em função da grande quantidade de nós normalmente existente nas peças dessa espécie, o que facilitou o estudo. Além disso, em peças comerciais é comum a presença de medula e madeira juvenil, devido ao pequeno diâmetro das toras em função da idade de corte. Para os ensaios foram utilizadas 180 peças de $0,027 \mathrm{~m}$ de espessura, $0,10 \mathrm{~m}$ de largura e $0,25 \mathrm{~m}$ de comprimento. As peças foram retiradas de tábuas comerciais adquiridas em serraria e ensaiadas na umidade de equilíbrio ao ar (aproximadamente $12 \%$ na região de Campinas).

Para a realização dos ensaios utilizou-se o equipamento de emissão de ondas de ultra-som marca Steinkamp BP-7, com transdutores de $45 \mathrm{kHz}$ e gel medicinal na interface transdutormadeira.

\section{Métodos}

As 180 peças foram submetidas à análise visual, por meio de inspeção visual, a qual constou deetalhamento de cada peça, indicando-se por peça sã aquela isenta de qualquer tipo de defeito; peças com desvio de grã aquelas que apresentavam inclinção grã superior a $6^{\circ}$; peças com nós e com presença de medula. Posteriormente, as mesmas peças foram submetidas ao ultra-som, no qual se mediu o tempo de propagação da onda para posterior cálculo da velocidade. Para a análise dos resultados, os dados obtidos da análise visual foram divididos em dois grupos: peças com defeito (D) e sem defeito (ND). Por não prejudicar significativamente a qualidade da madeira, as peças identificadas pela análise visual, apresentando somente pequenos desvios de fibra, foram consideradas não defeituosas (ND). Com esta caracterização, foram executados os Box-Plot, a análise do histograma e o teste de normalidade de Anderson Darling dos dois grupos, utilizando-se o MINITAB. Posteriormente, as médias de (D) e (ND) foram comparadas utilizando-se o teste não paramétrico, de Mann-Whitney. Visando-se a obtenção de intervalos de velocidade que caracterizassem a presença de defeitos, aplicaram-se modelos utilizando-se o programa SPSS. Finalmente, foram calculados os intervalos de confiança da velocidade para a média das peças com defeito. 


\section{RESULTADOS E DISCUSSÃO}

Os resultados obtidos pela análise visual e ensaio com ultrasom estão apresentados na Tabela 1.

$\mathrm{O}$ 'Box-Plot' indicou diferença significativa entre as duas medianas (5276 para D e 6510 para ND). O valor do terceiro quartil de $\mathrm{D}$ é menor que o do primeiro quartil do $\mathrm{ND}$, evidenciando a grande diferença entre os dois grupos. A amplitude entre os quartis é maior para o D (1185 contra 726.5 do ND). Observa-se, para os ND, quatro possíveis outliers, enquanto para os $\mathrm{D}$, apenas um.

Os histogramas para D e ND demonstraram que os valores do grupo D estão mais concentrados que os do grupo ND. Construindo-se o gráfico de normalidade para os dois grupos e se aplicando o teste de normalidade de Anderson Darling, demonstra-se que nas peças com defeito a hipótese de normalidade não foi rejeitada (valor $p=0,161$ ). Nas peças sem defeito, com um valor $\mathrm{p}<0,0001$, rejeitou-se a hipótese de normalidade.

Aplicando-se o teste de Mann-Whitnney para comparação dos dois grupos, obteve-se um valor para a estatística do teste $\mathrm{T}=3879.0$. A hipótese de igualdade de média para os dois grupos é rejeitada com um valor $\mathrm{p}<0,0001$. Com isto, tem-se mais um indicador forte de que existe diferença entre o grupo de peças com e sem defeito.

Para a aplicação do modelo logístico, foram consideradas duas variáveis - Dependente e Independente. Para a variável dependente (De) atribuiu-se valor 1 para peças com presença de defeito e valor 0 para peças sem defeito. A velocidade obtida por meio do ultra-som ( $\left(\mathrm{em} \mathrm{m} \mathrm{s}^{-1}\right)$ foi considerada variável independente.

Tabela 1. Observações da análise visual e resultados das velocidades obtidas utilizando-se equipamento de ultra-som

\begin{tabular}{|c|c|c|c|c|c|c|c|c|c|c|c|}
\hline Peça & $\begin{array}{c}\text { Análise } \\
\text { Visual }\end{array}$ & $\begin{array}{l}\text { Ultra-som } \\
\mathrm{m} \mathrm{s}^{-1}\end{array}$ & Peça & $\begin{array}{c}\text { Análise } \\
\text { Visual }\end{array}$ & $\begin{array}{l}\text { Ultra-som } \\
\mathrm{m} \mathrm{s}^{-1}\end{array}$ & Peça & $\begin{array}{c}\text { Análise } \\
\text { Visual }\end{array}$ & $\begin{array}{l}\text { Ultra-som } \\
\mathrm{m} \mathrm{s}^{-1}\end{array}$ & Peça & $\begin{array}{c}\text { Análise } \\
\text { Visual }\end{array}$ & $\begin{array}{l}\text { Ultra-som } \\
\mathrm{m} \mathrm{s}^{-1}\end{array}$ \\
\hline 2 & Desvio + nó & 5734 & 47 & Sem defeito & 6964 & 92 & Sem defeito & 5907 & 137 & Nó & 6010 \\
\hline 4 & Sem defeito & 6862 & 49 & Sem defeito & 6775 & 94 & Nó & 4721 & 139 & Medula & 4875 \\
\hline 5 & Desvio & 6739 & 50 & Sem defeito & 7123 & 95 & Nó & 4368 & 140 & Desvio & 6521 \\
\hline 6 & Desvio + nó & 5900 & 51 & Desvio & 7042 & 96 & Sem defeito & 5109 & 141 & Desvio & 6734 \\
\hline 9 & Sem defeito & 7230 & 54 & Desvio & 6868 & 99 & Sem defeito & 6328 & 144 & Desvio + nó & 5950 \\
\hline 10 & Desvio + nó & 6932 & 55 & Desvio & 7284 & 100 & Desvio & 6272 & 145 & Sem defeito & 6478 \\
\hline 11 & Nó & 3261 & 56 & Sem defeito & 7222 & 101 & Desvio & 6334 & 146 & Sem defeito & 6427 \\
\hline 12 & Nó & 2765 & 57 & Sem defeito & 6887 & 102 & Sem defeito & 5995 & 147 & Desvio + nó & 5900 \\
\hline 13 & Medula & 5473 & 58 & Sem defeito & 6782 & 103 & Desvio & 5480 & 148 & Sem defeito & 5750 \\
\hline 14 & Sem defeito & 3383 & 59 & Nó & 6024 & 104 & Desvio & 5796 & 149 & Desvio & 6726 \\
\hline 19 & Medula & 6870 & 64 & Desvio & 7032 & 109 & Medula & 5981 & 154 & Sem defeito & 6684 \\
\hline 20 & Nó & 5970 & 65 & Sem defeito & 6932 & 110 & Nó & 6010 & 155 & Sem defeito & 6925 \\
\hline 21 & Sem defeito & 6944 & 66 & Sem defeito & 7156 & 111 & Nó & 5511 & 156 & Sem defeito & 6775 \\
\hline 22 & Desvio & 6165 & 67 & Desvio & 6555 & 112 & Sem defeito & 5955 & 157 & Sem defeito & 6263 \\
\hline 23 & Desvio & 6609 & 68 & Sem defeito & 6266 & 113 & Sem defeito & 6203 & 158 & Nó & 5605 \\
\hline 24 & Sem defeito & 6906 & 69 & Sem defeito & 6631 & 114 & Nós & 6039 & 159 & Sem defeito & 6378 \\
\hline 25 & Sem defeito & 6915 & 70 & Sem defeito & 6637 & 115 & Desvio & 5610 & 160 & Sem defeito & 6143 \\
\hline 26 & Desvio + nó & 6849 & 71 & Nó & 6281 & 116 & Medula & 6005 & 161 & Sem defeito & 6474 \\
\hline 27 & Sem defeito & 7159 & 72 & Sem defeito & 6944 & 117 & Medula & 5317 & 162 & Nó & 4786 \\
\hline 28 & Desvio & 7418 & 73 & Desvio & 6925 & 118 & Medula & 4823 & 163 & Medula & 4409 \\
\hline 29 & Desvio & 7353 & 74 & Nó & 6487 & 119 & Nó & 4331 & 164 & Medula & 4983 \\
\hline 30 & Desvio + nó & 6438 & 75 & Sem defeito & 6638 & 120 & Medula & 5095 & 165 & Medula & 5187 \\
\hline 39 & Sem defeito & 6200 & 84 & Sem defeito & 6250 & 129 & Nó & 5271 & 174 & Medula & 5133 \\
\hline 40 & Sem defeito & 6148 & 85 & Sem defeito & 6376 & 130 & Medula & 5716 & 175 & Medula & 5102 \\
\hline 41 & Sem defeito & 6317 & 86 & Sem defeito & 6438 & 131 & Nó & 5059 & 176 & Medula & 5088 \\
\hline 42 & Sem defeito & 5244 & 87 & Sem defeito & 5924 & 132 & Desvio & 5697 & 177 & Medula & 5222 \\
\hline 43 & Sem defeito & 5207 & 88 & Nó & 5190 & 133 & Sem defeito & 6399 & 178 & Nó & 4189 \\
\hline 44 & Nó & 5464 & 89 & Sem defeito & 5869 & 134 & Sem defeito & 6510 & 179 & Medula & 5993 \\
\hline 45 & Nó & 6010 & 90 & Medula & 6094 & 135 & desvio & 6492 & 180 & Desvio & 6938 \\
\hline
\end{tabular}


Aplicando-se o modelo às variáveis definidas foram obtidos os seguintes resultados:

Modelo estimado:

$$
\log \operatorname{it}[\pi(\mathrm{v})]=\log \left(\frac{\pi(\mathrm{v})}{1-\pi(\mathrm{v})}\right)=12,4197-0,0021 \mathrm{v}
$$

Os resultados dos testes de significância do modelo foram: 'Goodness of fit' = 344,034; 'Log Likehood Ratio' = 163,502; $\mathrm{X}^{2}=81.007$ (valor $\mathrm{p}<0,0001$ ) e Razão de acerto entre observados e preditos: $78,89 \%$.

A significância dos parâmetros pode ser visualizada na Tabela 2.

Os valores obtidos nos testes de significância demonstram que o modelo é altamente significante. Com 79,89\% de acerto dos valores observados para os preditos, diz-se que o modelo tem poder de predição satisfatório.

Ambos os parâmetros e $\beta$ foram significativos para o modelo. Para o $\alpha$, a estatística do teste de Wald foi igual a 41,4454 , com valor $\mathrm{p}<0,0001$. Para o parâmetro $\beta$, a estatística do teste de Wald foi igual a 44,4983, com valor $\mathrm{p}<0,0001$. Pode-se concluir, portanto, que a variável v é altamente significativa para o modelo.

O valor da $\operatorname{Exp}(\beta)$ é igual ao incremento no 'odds' de presença de defeito para cada unidade acrescentada à variável independente. No modelo, o odds estimado de presença de defeito decresce $0,21 \%$ para cada unidade acrescentada a v.

$\mathrm{O}$ intervalo de confiança $(\alpha=0,10)$ para o parâmetro $\beta=-0,0021$ é :

$$
\begin{gathered}
{[-0,0021-1,64 \times 0,0003 ;-0,0021+1,64 \times 0,0003]} \\
{[-0,00259 ;-0,001608]}
\end{gathered}
$$

O intervalo de confiança $(\alpha=0,10)$ para o parâmetro $\alpha=12,4197$ é:

$$
\begin{gathered}
{[12,4197-1,64 \times 1,9292 ; 12,4197+1,64 \times 1,9292]} \\
{[9,255812 ; 15,583588]}
\end{gathered}
$$

A variável independente $\mathrm{v}$ apresenta uma grande dimensão, acarretando um número elevado de níveis, dificultando a interpretação da $\operatorname{Exp}(\beta)$ pois o incremento no 'odds' de presença de defeito é muito pequeno $(0,21 \%)$ para cada nível a mais de $\mathrm{v}$, esse problema pode ser resolvido categorizando-se a variável $\mathrm{v}$; para isto, a variável $\mathrm{v}$ foi dividida em dois intervalos, ou seja:

$$
Z=\begin{array}{lll}
0 & \text { se } & v \leq 6000 \mathrm{~m} \mathrm{~s}^{-1} \\
1 & \text { se } & v>6000 \mathrm{~m} \mathrm{~s}^{-1}
\end{array}
$$

O valor de corte foi adotado com base nas estatísticas descritivas, ou seja, foi calculada a média entre o limite superior da média do grupo D mais dois desvios padrão e o limite inferior da média do grupo ND menos dois desvios padrões.

Aplicando o modelo usando a variável dependente (De) e a nova variável independente $Z$ (categorizada), foram obtidos os seguintes resultados:

Modelo estimado:

$$
\log \operatorname{it}[\pi(\mathrm{v})]=\log \left(\frac{\pi(\mathrm{v})}{1-\pi(\mathrm{v})}\right)=1,0629-2,5215 \mathrm{v}
$$

Os resultados dos testes de significância do modelo foram: 'Goodness of fit' = 179,978; 'Log Likehood Ratio' = 186,978; $\mathrm{X}^{2}=57,531$ (valor $\mathrm{p}<0,0001$ ) e Razão de acerto entre observados e preditos: $78,33 \%$.

A significância dos parâmetros pode ser visualizada na Tabela 3.

Os valores obtidos nesse teste de significância indicam que o modelo é altamente significante. Com 79,33\% de acerto dos valores observados para os preditos, pode-se dizer que o modelo tem poder de predição satisfatório.

Ambos os parâmetros, $\alpha$ e $\beta$, foram significativos. Para o $\alpha$, a estatística do teste de Wald foi igual a 15,9538, com valor $\mathrm{p}<0,0001$. Para o parâmetro $\beta$, a estatística do teste de Wald foi igual a 48,0050 (maior que no modelo anterior, cujo valor foi $\mathrm{W}=44,4983$ ) com valor $\mathrm{p}<0,0001$. Pode-se concluir, então, que a variável $\mathrm{Z}$ é altamente significativa para o modelo.

Levando-se em conta o valor da $\mathrm{E}(\beta)$ pode-se concluir que, quando o valor da velocidade muda do intervalo $\mathrm{Z}<6000 \mathrm{~m} \mathrm{~s}^{-1}$ para o intervalo $Z>6000$, a propriedade de defeito decresce 91,97\%.

O intervalo de confiança $(\alpha=0,10)$ para o parâmetro $\beta$ é:

$$
\begin{gathered}
{[-2,5215-1,64 \times 0,3639 ;-2,5215+1,64 \times 0,3639]} \\
{[-3,118296 ;-1,924704]}
\end{gathered}
$$

é:

O intervalo de confiança para $(\alpha=0,10)$ para o parâmetro $\alpha$

\begin{tabular}{|c|c|c|c|c|c|c|}
\hline Variáveis & $\beta$ & Desvio & Wald & GL & Sig & $\operatorname{Exp}(\beta)$ \\
\hline $\mathrm{v}(\beta)$ & $-2,5415$ & 0,3639 & 48,0050 & 1 & 0,0000 & 0,0803 \\
\hline Constante $(\alpha)$ & 1,0629 & 0,2661 & 15,9538 & 1 & 0,0001 & \\
\hline
\end{tabular}

$$
\begin{gathered}
{[1,0629-1,64 \times 0,2661 ; 1,0629+1,64 \times 0,2661]} \\
{[0,626496 ; 1,499304]}
\end{gathered}
$$

Sendo assim, a probabilidade estimada de se ter uma peça defeituosa se a velocidade do ultra-som medida estiver contida no intervalo $\mathrm{Z}<6000$, é:

$$
\pi(Z=0)=\frac{\exp (1,0629-2,5215 \times 0)}{1+\exp (1,0629-2,5215 \times 0)}=0,7432=74,32 \%
$$

Tabela 2. Testes de significância do modelo

\begin{tabular}{ccccccc}
\hline Variáveis & $\beta$ & Desvio & Wald & GL & Sig & Exp $(\beta)$ \\
$\mathrm{v}(\beta)$ & $-0,0021$ & 0,0003 & 44,4983 & 1 & 0,0000 & 0,9979 \\
Constante $(\alpha)$ & 12,4197 & 1,9292 & 41,4454 & 1 & 0,0000 & \\
\hline
\end{tabular}

Tabela 3. Testes de significância do modelo utilizando a variável v categorizada 
Calculando-se a mesma probabilidade para os limites inferiores e superiores dos intervalos de confiança para $\alpha$ e $\beta$, tem-se o intervalo de confiança para $\pi(Z=0)$ :

Para o limite inferior:

$\pi(Z=0)=\frac{\exp (0,626496-3,118296 \times 0)}{1+\exp (0,626496-3,118296 \times 0)}=0,6517=65,17 \%$

Para o limite superior:

$\pi(Z=0)=\frac{\exp (1,499304-1,924704 \times 0)}{1+\exp (1,499304-1,924704 \times 0)}=0,8175=81,75 \%$

De forma análoga, é possível se calcular a probabilidade estimada de se ter uma peça não defeituosa, se a velocidade do ultra-som medida estiver contida no intervalo $\mathrm{Z}<6000$ :

$$
\pi(Z=1)=\frac{\exp (1,0629-2,5215 \times 1)}{1+\exp (1,0629-2,5215 \times 1)}=0,1887=18,87 \%
$$

Calculando-se os intervalos de confiança para $\pi(Z=1)$ :

$$
\begin{aligned}
& \pi(Z=1)=\frac{\exp (0,626496-3,118296 \times 1)}{1+\exp (0,626496-3,118296 \times 1)}=0,0764=7,64 \% \\
& \pi(Z=1)=\frac{\exp (1,499304-1,924704 \times 1)}{1+\exp (1,499304-1,924704 \times 1)}=0,3952=39,52 \%
\end{aligned}
$$

Como já foi demonstrado, os valores das velocidades para as peças defeituosas têm distribuição normal, sendo assim, é possível calcular o intervalo de confiança $(1-\alpha)$ para a média (variância desconhecida) da seguinte forma:

$$
\left[\overline{\mathrm{X}}-\mathrm{n}^{-1 / 2} \hat{\sigma} \times \mathrm{t}_{\mathrm{n}-1,1-\alpha / 2} ; \overline{\mathrm{X}}+\mathrm{n}^{-1 / 2} \hat{\sigma} \times \mathrm{t}_{\mathrm{n}-1,1-\alpha / 2}\right]
$$

Calculando-se o intervalo de confiança de $\alpha=0,10$ para a média das peças com defeito, tem-se:

$$
\left[5359,3-\frac{1}{\sqrt{180}} \times 843,1 \times 1,64 ; 5359,3+\frac{1}{\sqrt{180}} \times 843,1 \times 1,64\right]
$$

$$
[5259,3 ; 5462,36]
$$

Os testes estatísticos realizados nessa primeira etapa do trabalho não contemplaram questões ligadas às diferenciações de defeitos (nós, presença medula etc.), nem o detalhamento desses defeitos, tais como tamanho e posição dos nós, uma vez que o objetivo era avaliar-se, inicialmente, a sensibilidade do equipamento na detecção de algum tipo de defeito nas peças. $\mathrm{O}$ aprofundamento do estudo, com a avaliação dos intervalos de velocidade representativos de cada tipo de defeito, bem como a quantificação de qual defeito tem maior influência na velocidade de propagação das ondas é objetivo da próxima etapa, para a qual será necessária uma amostra maior do que a que foi utilizada nesse trabalho.

\section{CONCLUSÕES}

1. Comprovou-se estatisticamente que, em relação à velocidade de propagação da onda, o grupo de peças de madeira com defeitos foi significativamente diferente que o grupo de peças de madeira sem defeitos.

2. Foi possível estabelecer-se um intervalo de velocidade que, para as peças defeituosas, possibilita a chance de confirmação de defeito, de 74,32\%. Para as peças sem defeito, a chance de o ultra-som indicar que a peça é defeituosa, é de $18,87 \%$, ou seja, pode ser definido o seguinte critério:

$\mathrm{v}<6000 \mathrm{~m} \mathrm{~s}^{-1} \Rightarrow 74,32 \%$ chance de presença de defeito IC $(90 \%)=[65,17 ; 81,75]$

$\mathrm{v}>6000 \mathrm{~m} \mathrm{~s}^{-1} \Rightarrow 18,87 \%$ chance de presença de defeito IC $(90 \%)=[7,64 ; 39,52]$

\section{LITERATURA CITADA}

ABNT - Associação Brasileira de Normas Técnicas. NBR 9192/ 95 - Paletes de Madeira - Materiais. Rio de Janeiro, 1997. 6p.

Agresti, A. Categorical data analysis. New York: John Wiley. 1990.558p.

Bucur, V. La vitesse des ultrasons et la qualité du bois. In: Actas du Colloque du Groupe Français de Rhéologie, Paris, v.19, 15p, 1984.

Bucur, V. Acoustics of wood. New York: CRC Press, 1995. 284p.

Chazelas, J.L.; Vergne, A.; Bucur, V. Analyse de la variation des propriétés physiques et mechániques locales du bois autour des noeuds. In: Actes du Colloque Comportement Mecanique du Bois, Bordeaux, june, p.376-386, 1988.

Conover, W.J. Practical nonparametrics statistics. 2 ed. New York: John Wiley. 1980. 493p.

IBDF - Instituto Brasileiro de Desenvolvimento Florestal. Brasil. Norma para classificação de madeira serrada de folhosas. Ministério da Agricultura. 1983.67p.

Jayne, B.A. Vibrational properties of wood as indices of quality. Forest Products Journal, Madison, v.9, n.11, p.413-416, 1959.

McDonald, K.A. Lumber defect detection by ultrasonics. Forest Products Laboratory, Madison, Wis, 1978, 20p. Research paper FPL 311

Meyer, P.L. Probabilidade aplicações á estatística. Rio de Janeiro: Livros Técnicos e Científicos. 2 ed., 1983. 426p.

Mood, A.M. Introduction to the theory of statistics. New York: McGraw-Hill. 3 ed., 1974. 564p.

Sandoz, J.L. Grading of construction timber by ultrasound. Wood Science and Technology, New York, v.23, n.2, p.95-108, 1989.

Steiger, R. Mechanische Eigenschaften von Schweizer FichtenBauholz bei Biege-Zug, Druck- und kombinierter M/N Beanspruchung. Zürich: Eidgenössischen Technischen Hochschule, 168p. Doktorarbeit in Technischen Wissenschaften 